\title{
Die sozialen Kommunikationsmittel in Lateinamerika: Realität und Problematik
}

\author{
von der Gruppe APEC, Montevideo
}

\section{Kirche und Medien der sozialen Kommunikation: Lebraussagen}

Wir behandeln zunächst den Fragenkomplex, wie er sich auf theoretischer Ebene für die Gesamtkirche stellt: In einer ersten Etappe nahm die Kirche den Medien der sozialen Kommunikation (MSK) gegenüber eine negative Haltung ein. Sie betrachtete die Medien als profanes, dem Bereich Gottes fremdes Erzeugnis. Sie waren gefährlich und konnten in Irrtum und Verwirrung führen: man mußte sich gegen sie verteidigen, weil sie eng mit der Sünde verknüpft waren. In den lateinamerikanischen Seminarien wurde den künftigen Priestern ihr Gebrauch verboten.

Ein zweiter Abschnitt ist durch defensive Haltung gekennzeichnet. Angesichts der profanen Entwicklung der MSK wurde gefordert, daß die Kirche sich ihre eigenen Medien schaffe, um ihre Herde zu verteidigen. Es gab zahlreiche Bemühungen, Medien zu erwerben, um die Christen mit ihrer Hilfe zu führen. So entwickelten sich die "Gute Presse", die konfessionellen Radiostationen, die Kinosäle in den Pfarreien. In den Seminarien wurden eine ausgewählte Presse und bis zu acht Filme pro Jahr zugelassen.

Mit dem zweiten Vatikanum begann eine Aussöhnung. Die MSK wurden in einen breiteren Zusammenhang gesehen, in dem die Barriere zwischen Heiligem und Profanem außeracht blieb. "Gaudium et Spes“ sollte die letzten Schwierigkeiten ausräumen: die Bemühungen der Menschen, miteinander in Kommunikation zu treten, werden als Teil des Planes Gottes gesehen. Der Umschwung, den das Konzilsdokument über die MSK („Inter Mirifica“) brachte, war positiv und endgültig.

Da man jedoch den technischen Fortschritt in sich selbst betrachtet, nicht als Teil der Gesamtwirklichkeit, sondern davon unabhängig, wird die Problematik noch nicht genügend klar erkannt, die sich ergibt aus dem Funktionieren, den Besitzverhältnissen, der Manipulation und dem Inhalt der Medien. Von daher läßt sich auch die optimistische und rühmende Einstellung dieses Dokuments erklären und der Mangel an kritischer Haltung. Man muß dabei berücksichtigen, daß es sich hier um das erste Dokument des Konzils handelt, das verabschiedet wurde, da man gerade mit dem Schema 13 begonnen hatte, dessen reiche Problematik dem Mediendokument seine spezifische Qualität geben sollte. Die Pastoralinstruktion „Communio et Progressio" erscheint kritischer Betrachtung offener, ist der Problematik näher, die von der Gesamtwirklichkeit gestellt wird. wenngleich sie diese nicht eigentlich von den Wurzeln her angeht.

Diese verschiedenen Etappen müssen in ihrer Entwicklung mit anderen Uberlegungen und Dokumenten der Kirche zusammen gesehen werden. Die Fragen der sozialen, politischen und ökonomischen Beziehungen zwischen den Menschen und Völkern sind

In der APEC („Asociación para Emisiones culturales“, Montevideo, Uruguay) arbeitet eine Gruppe katholischer Rundfunkverantwortlicher für Lateinamerika zusammen. Mitglieder sind u. a. P. Manuel Olivera, UNDA-Verantwortlicher für Lateinamerika und Vizepräsident von UNDA-Welt, und Mario Kaplun. 
mit großem Nachdruck behandelt in Mater et Magistra, Populorum Progressio, Octogesimo Adveniens usw. Hier wird eine kritische Einstellung der Kirche gegenüber den Strukturen deutlich, gegenüber ökonomischer Macht, internationalem Handel, kapitalistischem Liberalismus usw. Erst durch diese Dokumente wird ein kritisches Urteil über die MSK möglich, wenngleich sie dort nicht ausdrücklich erwähnt sind. Für die lateinamerikanische Kirche des Jahres 1968 erarbeitete Medellín sein Dokument über die MSK. Es steht in der Linie von "Inter Mirifica" mit seiner positiven Sicht und der durchwegs erkennbaren Grundeinstellung, daß die Medien autonom und von den konkreten Bedingungen unabhängig sind. Zwar werden einige Einschränkungen und Probleme aufgezeigt, doch stehen sie nicht im Vordergrund und werden nicht weiter aufgearbeitet.

In anderen Dokumenten von Medellín wird verlangt, daß die Medien bewußtseinsändernde Funktionen haben und sich in den Dienst der Strukturveränderung, notwendiger und tiefgreifender umfassender Veränderungen stellen sollen, wie sie die lateinamerikanischen Bischöfe forderten. Diese Aussagen eröffnen einen neuen, originalen und interessanten Weg für die Arbeit. Es gab in Medellín auch noch andere Überlegungen, die die spätere Interpretation der MSK, die die lateinamerikanische Kirche erarbeitete, sehr beeinflußten. Wir erwähnen zwei:

Der Gedanke der Befreiung (liberación) wird zu gegebener Zeit von Bedeutung sein bei der Frage, ob die MSK wirklich befreien oder versklaven. Das Konzept einer befreienden Erziehung wird die Frage aufwerfen, ob die MSK tatsächlich für dieses Ziel eingesetzt werden können oder schon eingesetzt wurden.

Zugleich entwickelten Fachleute andere Überlegungen, die im kirchlichen Raum auf diesem Gebiet wichtige und besondere Beiträge lieferten. Die Schaffung einer Abteilung MSK innerhalb des CELAM ${ }^{1}$ und die Beiziehung von Experten in diesem Gremium hatte einen entscheidenden Einfluß. Das Seminar von Santa Inés brachte 1966 eine Kerngruppe von zahlreichen katholischen Kommunikatoren zusammen, die erstmals die Situation der Medien in der Gesamtwirklichkeit untersuchte, was sie sind und wie sie funktionieren.

Zwei spätere Seminare vertieften und verschärften diesen Gesichtspunkt: das der UNDA/AL und des Radiodienstes von Sutatenza 1970, und das von Mexico über Erziehung und soziale Kommunikation 1971. Hier werden die MSK nicht mehr als ideale Plattform gesehen, sondern in der Alltäglichkeit ihrer Arbeit betrachtet. Diese Betrachtungsweise, die wir realistisch nennen möchten, bestimmte auch die regionalen Treffen, die DECOS ${ }^{2}$ im Jahre 1972 über die MSK veranstaltete und deren Leitlinien von ihrer eigenen Abteilung dem CELAM 1973 als grundlegende pastorale Kriterien für die Arbeit der Evangelisation in den MSK vorgeschlagen wurden.

\section{Die Wirklichkeit der MSK in Lateinamerika: Historisch-kritis he Übersidht}

Wir wollen in Kürze diese realistische Sicht darstellen, die die MSK innerhalb der anderen Faktoren sieht, die das lateinamerikanische Leben bestimmen. Die folgende Frage kann das Problem verdeutlichen: Warum erfüllen die MSK nicht die Funktion positiver Triebkräfte, die ihnen Medellín zuschreibt? Oder anders: Sind die Medien vom Gesamtsystem unabhängig genug, daß sie dieses System in Frage stellen und die Entwicklung einer neuen, anderen Gesellschaft fördern können? Beachten sie den 
Rahmen der notwendigen ideologischen, politischen und ökonomischen Unabhängigkeit? Anhand der Bedingungen, wie sie verschiedene Studien in Lateinamerika erarbeitet haben, wollen wir auf diese Fragen Antworten zu geben versuchen.

\section{A. Strukturelle Bedingungen}

Die große Mehrheit der MSK befindet sich in den Händen privater Unternehmen mit kommerziellem Charakter, deren Hauptziel der Gewinn ist. Ganz anders ist das System in vielen europäischen Ländern - zum Beispiel bei Hörfunk und Fernsehen -, wo die Sender Eigentum des Staates sind und ihre Kanäle vor allem kulturellen und sozialen Fragen öffnen.

In Lateinamerika, das den liberalen Kriterien der USA folgt, sind die MSK vor allem Mittel, Produkte zu verkaufen; das System ist so organisiert, daß alle anderen Funktionen dieser einen untergeordnet sind. Nur der Film entzieht sich dieser Bedingung. Folglich arbeiten die MSK nicht für das Publikum, sondern für die Verbraucher, und diese Masse potentieller Verbraucher wird $\mathrm{zu}$ ihrem wichtigsten Adressaten.

Wenn es keinen möglichen Käufer für die angepriesenen Produkte gibt, besteht natürlich auch kein Interesse daran, neue Mediendienste zu schaffen. Daher die Konzentration der MSK in den städtischen Bezirken und die Tatsache, daß die Aufmerksamkeit sich vor allem auf das Publikum mit großer Kaufkraft richtet. Gewöhnlich sind die lateinamerikanischen MSK in wenigen Händen konzentriert.

Wenn es auch theoretisch eine Gleichheit der Möglichkeiten gibt, so haben doch nur Unternehmen mit großer ökonomischer Macht die Voraussetzungen, sie auszunutzen. Je komplizierter und kostspieliger die Medien werden, je mehr sie nach technischer Perfektion suchen und die hohe Auflage anstreben, um wirksam im Wettbewerb mithalten zu können, desto mehr konzentriert sich ihr Besitz bei denen, die zu ihrer Unterhaltung in der Lage sind. Gewiß werden Aktien verteilt und kommen Anzeigen herein, aber letztlich kann das doch nicht darüber hinwegtäuschen, daß die Konzentration in wenigen Händen voranschreitet.

Hier wird auch die enge Bindung zwischen Anzeigengeschäft und kommerziellem System, zwischen Industrie und MSK deutlich. Wenn wir eine Zeitung kaufen, so unterhält nicht unser Geld das Unternehmen, wir können nicht einmal die Papierkosten aufbringen. Die Zeitungen werden direkt von der Werbung bezahlt und indirekt von der Industrie und dem Handel, der sie fördert. So sind die Medien einmal abhängig von mächtigen Unternehmen und zum anderen von den Anzeigenkunden.

Die quantitative und qualitative Unterentwicklung der MSK in Lateinamerika wird also nicht verursacht durch den Mangel an Publikum, sondern durch den Mangel an Verbrauchern, das heißt einem Publikum mit Kaufkraft. Wir haben gewaltige verarmte Massen, die die MSK als Hilfe brauchen; da diese Massen aber keine Kaufkraft haben, besteht kein Interesse, sich ihnen zuzuwenden und etwas für sie zu tun.

Solche Bedingungen schaffen den Menschen der Konsumgesellschaft, der nach Populorum Progressio gekennzeichnet ist durch seinen Wunsch "mehr zu haben ", nicht "mehr zu sein ${ }^{\circ}$. Das wird noch unterstützt durch ein Grundprinzip der Werbung, nach dem man an die Primärinstinkte appellieren muß, vor allem an den Egoismus 
des Menschen. So werden individualistische und materialistische Tendenzen gefördert, wird der Blick ausschließlich gerichtet auf wirtschaftlichen Aufstieg und den Erwerb neuer Güter, auch wenn sie überflüssig sind.

Diese Bedingungen, die wir nur skizziert haben, sind nicht nur sehr mächtig, sondern verdrängen auch das Verantwortungsgefühl und den guten Willen des Kommunikators. Es gibt Fälle von Medien, die mit guten Absichten begannen, dann aber ihre Arbeit einstellten oder sich den Strukturen anpaßten, die ihnen - entsprechend den strengen Normen des Systems - ihre Existenz ermöglichten. Der Appell an den guten Willen und die Ehrlichkeit der Eigentümer erweist sich von daher als ungenügend; und natürlich auch der Appell - bei verschiedenen katholischen Unternehmern - an die Prinzipien des christlichen Lebens, nach denen sie sich, auch im professionellen Bereich, richten sollen. Die Spielregeln sind schließlich stärker. Die Ausnahmen bestätigen nur die Regel.

Eine interessante Variante ist seit kurzem in Peru zu beobachten, wo ein Radio- und Fernsehgesetz einen tiefgreifenden Wandel der Strukturen dieser Medien bewirkt hat, weil der Staat die Mehrheit der Aktien übernahm. Diese Veränderung aber ist noch zu neu, um Gültiges über ihre Folgen sagen zu können.

\section{B. Politische Bedingungen}

Verschiedene Soziologen gehen so weit, daß sie in ihrer Analyse und Beschreibung der lateinamerikanischen MSK die Medien als Herrschaftsinstrumente der herrschenden Klasse sehen. Nach ihren Untersuchungen zwingen die Medien eine bestimmte Weltsicht auf, die jene der Herrschenden ist, mit dem Ziel, deren Macht weiter zu erhalten. Diese Behauptung wird in etwa durch die Analyse der Informationen gestützt, die fortwährend Tatsachen vertuschen oder verdrehen. Die Autoren weisen nach, daß sich in den letzten Jahren keine Entwicklung zu einer breiteren, objektiveren Information nachweisen läßt, die Situation in diesem Bereich sich vielmehr sehr verschlechtert habe: es seien gar Werte wie Ehrenhaftigkeit, die für die liberale Ideologie typisch seien, verschwunden. Es gehe darum, eine Schlacht zu schlagen, bei der alle Waffen gut sind, auch wenn man zu Lüge, Vertuschung oder Entstellung greifen müsse. Diese Autoren unterstreichen, daß es in Lateinamerika noch wenige Länder gebe, in denen Pressefreiheit existiere, auch wenn diese die relative und illusorische Pressefreiheit des Liberalismus sei.

Eine eiserne Zensur - manchmal offenkundig, manchmal verhüllt - kontrolliert und knebelt in fast allen Nationen unseres Subkontinents Information und Meinungsäußerung. Man weist auch hin auf die gefährliche Tendenz, die sich daraus ergibt, daß man Nachrichten in einer Informationsflut präsentiert, ein bei Radio und Fernsehen deutlich feststellbares Phänomen. Nicht genügend in Rechnung gestellt werde auch die rechte Gewichtung und Plazierung der Information, die es dem Empfänger gestatten, eine Nachricht einzuordnen. Die reichhaltige und bunt gemischte telegrammartige Kost bewirkt, daß eine an sich gute Information völlig ungenießbar wird und den Empfänger oder Leser noch mehr desorientiert und desinformiert, weil die Nachrichten nicht das Gewicht und den Platz erhalten, den sie im Gesamtrahmen des Lebens einnehmen.

Einige Autoren weisen auch darauf hin, daß die MSK sich nicht damit zufrieden geben, Nachrichten zu vermitteln, sondern selbst Nachrichten produzieren mit jenen 
effekthaschenden, sensationellen Methoden, die die Wirklichkeit zerstückeln. Sie betonen, daß auf diese Weise beim Volk nicht die Bewußtseinsbildung bezüglich der gemeinsamen wichtigen Probleme gefördert werde und auch nicht die Verantwortung, diese Probleme zu lösen. Es würden keine Hilfen geboten, die das Publikum instand setzten, bewußt und persönlich zu handeln. Im Gegenteil, eine Minderheit mache ihre Anschauungen verpflichtend und herrsche auf diese Weise weiter über die naiven Mehrheiten. Die Autoren finden ihre Ansicht bestätigt durch die Tatsache, daß die Medien nicht zur Bildung der öffentlichen Meinung beitragen, sondern insbesondere dem Eskapismus und der Unterhaltung dienen, vor allem im Fernsehen, Radio und kommerziellen Kino. Aber auch für die Zeitungen weisen sie nach, daß die Abteilung Unterhaltung immer mehr die der Information und Meinungsäußerung verdrängt. Sie weisen zudem darauf hin, daß bei den gedruckten Medien Bildillustrierte, Fotoromane, Comics usw. die größte Auflage und Verbreitung in Lateinamerika haben und - nach der treffenden Formulierung von Adorno - eine echte "Kulturindustrie" darstellen, die entfremdet und eine unkritische Gesellschaft erzeugt, jene „Gesellschaft ohne Opposition“, von der Marcuse spricht.

\section{C. Äußere Bedingungen}

Die lateinamerikanischen MSK befinden sich in einer Situation der Abhängigkeit und des Neokolonialismus, sind Opfer einer kulturellen Invasion, die die einheimischen Werte deformiert. Ihre einseitige Weltsicht führt dazu, die aus hochindustrialisierten Ländern übernommenen Modelle nachzuahmen. Es wird suggeriert, daß es nützlich und notwendig sei, wenn einige Länder als die Inkarnation des Guten dominieren, weil wir durch sie Leben, „Demokratie“ und Möglichkeiten der Weiterentwicklung erhalten. Es genügt der Hinweis, daß alle Information über das Weltgeschehen, Lateinamerika eingeschlossen, von ausländischen Agenturen kommt. Zwei von diesen, Associated Press und United Press, monopolisieren fast $80 \%$ aller Nachrichten, die in Lateinamerika verbreitet werden.

Die neuen Technologien, auch der wachsende Gebrauch von Kommunikationssatelliten, verbessern die Situation nicht; im Gegenteil, sie verschlechtern die Lage, weil nur Unternehmen mit großer ökonomischer und technologischer Kapazität in der Lage sind, diese Vorteile zu kontrollieren. Trotz der abgegebenen Kulturmanifeste wird nicht gesehen, daß die herrschenden Interessen in Wirklichkeit von anderer Art sind.

\section{Ideologische Bedingungen}

$\mathrm{Zu}$ den schon angeführten bewußten oder intendierten Bedingungen kommt die ideologische Kondition. Wenn auch von anderer Art, widerspricht sie dem bisher Gesagten nicht, sondern bestätigt es auf einer grundlegenden Ebene. In diesem Bereich hat in den letzten Jahren die Wissenschaft, ausgehend von Semiologie und Semiotik, wertvolle Beiträge geliefert. Dank dieser neuen Wissenschaften konnten bestimmte Empirismen überwunden, konnte objektiv festgestellt werden, bis zu welchem Grade die MSK bestimmten Ideologien entsprechen, die sie bedingen. Eindeutiger als früher konnte festgestellt werden, welchen Einfluß Ideologie - unabhängig von der Absicht des Senders - hat. Auf diese Weise haben verschiedene 
Autoren die Botschaften der Medien analysiert und die darin enthaltene herrschende Ideologie dekodiert.

Aus solcher Analyse erwuchsen wichtige Interpretationen:

- Manche sehen die Beziehung zwischen MSK und Publikum als Interaktion. Die Medien spiegeln, nach dieser Sicht, was das Publikum denkt, fühlt und wünscht. Sie geben dem Publikum ein Bild seiner selbst. Es gibt ein fortwährendes Feedback zwischen den Wünschen des Publikums und den Inhalten der Medien.

Auch einige marxistische Analytiker geben zu, daß die MSK nicht Ideologie erfinden oder schaffen. Sie verbreiten, konsolidieren und fixieren aber eine Ideologie, geben ihr den Vorzug vor anderen möglichen, wobei sie sich auf Ideen, Vorstellungen und Vorurteile stuitzen, die schon im Volk vorhanden sind. Mattela etwa kommt zu dem Schluß, wenn auch seine Úberlegungen nicht immer folgerichtig sind, daß die herrschenden Klassen nicht Mythen schaffen, sondern sie verwalten; diese Mythen bestehen schon in den Volksmassen.

- Eine andere Meinung behauptet, daß die MSK eindeutig manipulieren. Sie kümmern sich nicht um das, was das Publikum denkt, sondern verbreiten bestimmte Wunschvorstellungen und versuchen ihnen Geltung zu verschaffen, wobei sie eine hohe Wirksamkeit erreichen. Das heißt: das Publikum kommt schließlich soweit, diese Vorstellungen für seine eigenen zu halten, obwohl es tatsächlich nicht so ist.

Das Publikum internalisiert die Einflüsse und Werte, die ihm vorgelegt werden, und reagiert freiwillig in der vorgegebenen Richtung. Um eine Formulierung von Paulo Freire zu gebrauchen: „Der Unterdrücker introjiziert seine Ideologie in den Beherrschten." Es steht uns nicht an, festzulegen, welche dieser Positionen am meisten zutrifft, möglicherweise enthalten beide Wahrheitselemente.

Andererseits muß darauf hingewiesen werden, daß diese semiologische Analyse einen neuen und wichtigen Weg eröffnet: in jeder der beiden Interpretationen wird für die befreiende Kommunikation ein kultureller Wandel als notwendig vorausgesetzt. Sie zeigt sowohl die große Schwierigkeit dieser Aufgabe als auch Wege, wie das Werk angegangen werden kann. Es handelt sich nicht nur darum, die Ausdrucksweise zu ändern, sondern $\mathrm{zu}$ einer Veränderung der Einstellung zu gelangen. Wenn die Medien, so wie sie derzeit gebraucht werden, einen konformistischen, passiven und individualistischen Menschen schaffen, genügt es nicht, diesem Menschen neue Begriffe zu vermitteln, vielmehr muß ihm die Möglichkeit gegeben werden, ein kritisches Bewußtsein zu gewinnen, das ihn zu einem aktiven, gesellschaftsfähigen und solidarischen Menschen umformt.

Solch kultureller Wandel setzt einen Menschen voraus, der fähig ist, sich von dem vermassenden Einfluß der Medien unabhängig zu machen. Dafür muß er sie kennen, sich mit ihnen beschäftigen, wissen, wie sie funktionieren, muß lernen, die verborgenen Ideologien, mit denen sie das Publikum fortwährend bombardieren, zu entdecken und zu dekodieren. Er muß die Manipulationsmechanismen und die Wirkungen, die sie erzeugen, kennen. Das erfordert praktischen Umgang mit den Medien beim einzelnen wie beim Volk, nicht mit dem Ziel, Spezialisten auszubilden, sondern um das Bewußtsein dafür zu schaffen, was die Medien sind und wie sie funktionieren.

Es wird immer deutlicher, daß ein Wandel dieser Art in der gegenwärtigen Welt sich nicht ohne die Medien vollziehen kann. Wenn diese Entwicklung nicht mit einer gewissen elementaren Praxis der Kommunikationstechnik verbunden ist, werden die großen und machtvollen Medien auch eine Gruppe leicht überspielen und sie mit ihrer 
massiven ideologischen Botschaft zudecken. Das bedeutet: es kann nicht nur darum gehen, sich der Medien zu bedienen, man muß sie auch als Gegenmittel benutzen. Das ist nicht leicht. Denn die Medien sind gewohnt, nicht nur von außen ihre Botschaft aufzudrängen, sondern sie rechnen auch mit der Komplexität der Eigenschaften, Tendenzen und Handlungen des Menschen, die seine egoistischen Instinkte verstärken. Dies ist die alte Sünde in immer neuen Kleidern. Der Kampf um die Verbesserung der Medien wird so zu einer neuen Form der Auseinandersetzung mit dem Fehler, der durch die herrschenden Zustände bedingt ist und die weniger edlen Tendenzen des Menschen stärkt.

\section{Die lateinamerikanische Kirche und die MSK: Konkrete Gesichtspunkte}

Zur Beurteilung der lateinamerikanischen Kirche in den MSK kann nicht auf Untersuchungen und Studien zurückgegriffen werden, die für eine objektive Wertung erforderlich sind. Obwohl die Forderung nach solchen Untersuchungen der "katholischen “ MSK Lateinamerikas schon seit langem erhoben wird, steht diese Arbeit noch aus. Folglich können nur Urteile formuliert werden, die - als Arbeitshypothesen der Betrachtung des Lesers überlassen bleiben.

Allgemein läßt sich sagen, daß die kircheneigenen Medien unter den systembedingten Verhältnissen leiden. Viele begannen mit guten Vorsätzen, aber nach einiger Zeit ließen kommerzielle Uberlegungen sie scheitern oder verwandelten sie um des Überlebens willen in Medien, die denen ähnlich waren, die zu bekämpfen man sich als Ziel gesetzt hatte. So wurden die guten Vorsätze, derentwegen diese Medien gegründet worden waren, praktisch zur Wirkungslosigkeit verdammt.

Die semiologische Analyse der Inhalte macht deutlich, daß die Mehrheit der Medien faktisch den Status quo festigt und damit im Gegensatz steht zu den von den lateinamerikanischen Bischöfen in Medellín formulierten Forderungen. Die behauptete religiöse Neutralität unterstïtzt natürlich das System und die von ihm verbreiteten Werte.

Weiter gibt es eine Haltung, die wir aktivistisch nennen könnten: man fordert die Präsenz der Kirche in den Medien ohne Rücksicht darauf, ob die Inhalte und Zeugnisse dem Evangelium entsprechen. Häufig werden die so produzierten Botschaften nicht nur zu völliger Wirkungslosigkeit verurteilt, sondern sie erreichen das genaue Gegenteil des erstrebten Ziels. Es gibt nur wenige, deren Arbeit es verdient, untersucht, verbreitet und nachgeahmt zu werden.

Man kann Begeisterung feststellen über den Gebrauch neuer Ausdrucksweisen, eine rein äußere Modernisierung, die häufig nicht begleitet wird von der Sorge, im Empfänger eine persönliche, bewußte, kritische und fragende Haltung zu erzeugen.

Die nichtkirchlichen Medien verhalten sich in den letzten Jahren der progressiven Kirche gegenüber feindlich: sie wird herabgesetzt, ihre Gedanken werden ignoriert, entstellt oder heftig angegriffen. Häufig wird der Begriff „subversiv“ suggeriert. Die von Medellín begonnene Bewegung findet die Tore der Medien verschlossen; es genügt an die Erklärungen und Manifeste der brasilianschen Bischöfe zu denken, deren Verbreitung in Brasilien verboten ist; ein ernsthaftes Problem, das die Kirche aufmerksam verfolgen muß. 
Bei den Christen gibt es verschiedene Haltungen bezüglich des Gebrauchs der MSK für die Evangelisation. Denjenigen, die die Medien für die besten modernen Mittel der Evangelisation halten, stehen andere gegenüber, die Zweifel anmelden und nur beschränkte Möglichkeiten sehen. Das Problem muß noch einmal durchdacht werden mit den Vorschlägen und Uberlegungen, die DECOS erarbeitet hat. $\mathrm{Zu}$ den Funktionen, die die lateinamerikanische Kirche übernehmen müßte, gehört die Anklage. Den spezifischen Charakter gibt ihr ihre prophetische Mission. Es muß analysiert werden, ob sie - im Bereich der kollektiven Kommunikation - diese Aufgabe wirkungsvoll erfüllt, und welche Wirkung erreicht wird. Es müßte auch untersucht werden, ob die Kirche in der lateinamerikanischen sozialen Kommunikation jene Aufgabe erfüllt, die ihr wiederholt zugeschrieben wurde, dort „Stimme derjenigen zu sein, die keine Stimme baben".

Nachdrücklich wird darauf verwiesen, daß der Kirche die schwierige Aufgabe der Erzieherausbildung zufalle, wobei aber vor allem auch der Inhalt dieser Ausbildung beschrieben werden muß. DECOS hat sie definiert mit dem Hinweis, daß es grundlegend darum gehe, ein kritisches Bewußtsein zu formen, nicht nur gegenüber den Medien, sondern auch gegenüber der Gesamtwirklichkeit, in die die Medien eingebettet sind. Es bleibt abzuwarten, mit welchen Mitteln, mit welcher Kraft, Intensität und Wirkung diese Aufgabe erfüllt wird.

Es gibt Diskussionen und Auseinandersetzungen über die Einschätzung der MSK durch den Christen. In diesem Zusammenhang ist die Spannung kennzeichnend, die 1971 im Seminar von Mexico entstand. Die Problematik zentriert sich auf verschiedene Punkte: Einmal geht es um die historischen Bedingungen. Während ihnen einerseits keine Bedeutung zugemessen wird und die Medien als Erzeuger der Veränderungen gesehen werden, werden sie andererseits als Teil der Gesamtwirklichkeit betrachtet und als von dieser konditioniert.

Unterschiedliche Auffassungen gibt es auch bezüglich der sinnenhaften, nicht rationalen Wahrnehmung, wie sie von den MSK gefördert wird. Die einen sehen dies als einen Fortschritt, eine Bereicherung des Menschen, die anderen weisen hin auf die mögliche Folge, daß die Fähigkeit zur Kritik dadurch geschwächt wird, weil die Fähigkeit zu überlegen und nachzudenken - grundlegende Bedingung jeder historischen Befreiung - abgebaut werde. Die beiden Positionen haben unterschiedliche Vorstellungen des "neuen Menschen“. Die eine glaubt, daß die Technologie, vor allem die elektronische, einen besseren, vollendeteren, mehr in die Welt integrierten und sozialeren Menschen schaffe, die andere hat dieser Sicht gegenüber Bedenken und geht - bei ihrem Konzept des „neuen Menschen “ - auf den heiligen Paulus zurüick, mit dem deutlichen Wunsch, das wesentlich Christliche klarer herauszustellen. Vielleicht wäre es gut, diese Diskussion weiterzuführen und zu vertiefen, damit man erkennt, ob beide Positionen voneinander unabhängig sind, sich ergänzen oder sich als miteinander unvereinbar erweisen.

\section{Die soziale Kommunikation und die Befreiung}

Wir wollen zum Schluß hinweisen auf die grundlegenden Forderungen, die nach Ansicht einiger Fachleute erfüllt sein müssen, wenn die Medien positive Faktoren der Veränderung sein sollen. Zunächst müßten Medien dieser Art den nationalen und regionalen Interessen dienen, sodann müßten sie es den Volksgruppen gestatten, sich in den Medien auszudrücken. 
Das geschieht aber nicht von selbst und auch nicht allein durch guten Willen, sieht man von den bescheidenen Ausnahmen ab, die die Regel bestätigen. Es ist auch kaum möglich innerhalb einer Struktur wie der gegenwärtigen. Damit die großen Medien auf diese Linie gebracht werden können, sind tiefgreifende strukturelle Veränderungen nötig.

So sagt man, drittens, daß die Medien die Gesellschaft nur in dem Maße verändern werden, wie sie sich selbst ändern. So wie das System insgesamt Möglichkeiten für die Befreiung des Menschen ermöglicht, werden Medien dieser Art entstehen. Anders bleiben sie Gefangene einer kommerziellen Struktur, und die Hoffnung auf diesen Wechsel ist utopisch.

Die gleiche Problematik bestimmt gegenwärtig den Bereich der Erziehung. Gegenüber denjenigen, die hoffen, daß die Erziehung die Gesellschaft verändere, stehen diejenigen, wirklichkeitsnäher, die darauf hinweisen, daß solche Umwandlung sich nicht vollziehen kann, wenn nicht das gesamte System sich ändert: die Veränderung des Systems erst werde eine neue Erziehung erlauben. Dies bedeutet keine passive Haltung, sondern meint, daß von den Medien allein oder von der Erziehung allein der Wechsel nicht zu erwarten ist. Deshalb wird, viertens, gefordert, die Medien als Beschleuniger dieser Gesamtveränderung zu benutzen, die wiederum echte Kommunikationsmedien ermöglichen wird. Bei der Frage danach, wie diese künftigen Medien funktionieren müßten, damit sie wirklich befreiend wirken können, ergeben sich neue Einsichten, aber auch große Schwierigkeiten.

Die bloße Veränderung von Strukturen genügt nicht. Die Medien könnten noch mehr entfremden und manipulieren als die gegenwärtigen. Oder wie es bei einem Regionaltreffen des DECOS ausgedrückt wurde: Strukturveränderung ist eine notwendige Bedingung, genügt aber nicht.

Ebensowenig ist der bloße Austausch von Inhalten ausreichend. Es geht nicht darum, einen Slogan durch einen anderen zu ersetzen, so progressiv er auch sei. Es gilt, die innere Struktur der Medien zu verändern. Diese Struktur besagt, daß die Medien heute Medien der Einbahnstraße, nicht aber des Dialogs sind. Kommunikation geschieht nur in eine Richtung. Die Medien sind in Wahrheit nur gewaltige Lautsprecher, durch die einige Wenige zu den Übrigen sprechen.

Nach Ansicht dieser Experten werden die Medien, solange sie einbahnstraßig bleiben und nur wenigen gestatten zu reden, während die große Masse passiv empfängt, Unfreiheit erzeugen und eine konformistische Gesellschaft stützen, wo die Vielen die Sicht aufnehmen, die andere ihnen vermitteln und wo die Medien anstelle der Massen die Wirklichkeit erfassen und denken.

Von daher sollte auch der Begriff „soziale Kommunikation“, auch wenn er nur beschreibende Funktion hat, kritisch befragt werden, weil er den Medien Möglichkeiten zugesteht, die sie nicht haben. Gegenüber dieser falschen Kommunikation und das wäre der fünfte Aspekt - sucht man nach Medien, die dialogische und mitbestimmende Kommunikation ermöglichen, d. h. Medien, die eine umfassendere, wirksamere und realere Teilnahme gestatten.

Schließlich sagen die Fachleute, daß diese dialogische Kommunikation ausgerichtet sei auf die Bildung des kritischen Bewußtseins als Mittel für die Befreiung und zugleich eines ihrer Ziele, weil der Mensch, der sich dieses kritische Bewußtsein 
erwerbe, schon dabei sei, sich zu befreien. Er bleibt nicht mehr passiv, sondern nimmt den Teil der Verantwortung auf seine Schultern, der ihm in der Gesellschaft zukommt.

\section{S UM M A R Y}

The problems of social communications in Latin America are considered by this group of Latinamerican Church media specialists against the background of the general situation of Church media, past and present. Despite the more positive basic attitude of the Conciliums "Inter Mirifica" and "Communio et Progressio", some parts of the documents of Medellin which concedes to the media an autonomous stand, and others who see the media in a capacity to change behaviour and structures, the real situation in Latin America is described in a pessimistic picture. The media are still quite dependent on cultural influences from overseas. On the other hand, media represent very often the ideology of less openly prevailing oppressors who might be anti-Church-oriented. Much has to be done for the Media in Latin America by the Church in order to come to a real communications dialogue fostering critical behaviour liberating man and helping towards his responsibility in society.

\section{RESUMEN}

Teniendo como telón de fondo la situación general de los medios de comunicación social, tal como la Iglesia católica los juzgó en el pasado y tal como se enfrenta a ellos en la actualidad, se desarrolla la problemática de la comunicación social en Latinoamérica partiendo de la realidad. A pesar de la actitud positiva de la Iglesia en los documentos conciliares "Inter Mirifica" y "Communio et Progressio», a pesar del documento de Medellín sobre los medios de comunicación social, que concede una posición autónoma a estos medios, a pesar de otros documentos de Medellín, que prescriben a dichos instrumentos de comunicación la función de mentalizar y promover el cambio de estructuras, se presenta sombría la situación real en Latinoamérica a tenor de los estudios de que se dispone. Los medios de comunicación social son víctima en alto grado de una invasión cultural llegada al subcontinente desde ultramar. Por lo demás son instrumento de la ideología de opresores, que además son enemigos de la lgelsia en múltiples aspectos. Por parte de la Iglesia debe hacerse mucho en favor de la comunicación social en Latinoamérica. Debe invertirse en ellos a fin de llegar a una verdadera comunicación dialogante que promueva la conciencia crítica, libere al hombre y le permita a la Iglesia cumplir con sus oblioaciones en la sociedad.

\section{RESUME}

A l'arrière-plan de la situation générale des mass-media, comment l'Eglise catholique les jugea dans le passé et comme elle se range à ses côtés maintenant, ainsi se dévoloppe la problématique de la communication sociale en Amérique Latine, d'après l'image de la réalité. En dépit de l'attitude fondamentale plus positive de l'Eglise dans les documents conciliaires «Inter Mirifica» et «Communio et Progressio», en dépit du document de Medellín qui concède aux mass-media une position autonome, en dépit aussi d'autres documents de Medellín qui attribuent aux mass-media des fonctions transformatrices de consciences et de structures, en dépit de tout cela la situation réelle en Amérique Latine est, en raison des recherches présentes, qualifiée de sombre. Les mass-media sont à une grande échelle les victimes d'une invasion culturelle, venue d'outre-mer, dans le subcontinent. Du reste, elles transmettent l'idéologie d'oppresseurs moins dominants qui sont par-dessus le marché encore sous plus d'un rapport anticléricaux. De la part de l'Eglise, il y a encore beaucoup à faire et à investir dans les mass-media en Amérique Latine afin d'arriver $a$ une communication véritablement en forme de dialogue qui favorise l'esprit critique, qui libère l'homme et lui fait percevoir sa responsabilité dans la société. 